\title{
Resection of gallbladder polyps following endoscopic ultrasound-guided cholecystoduodenostomy using a lumen-apposing metal stent
}

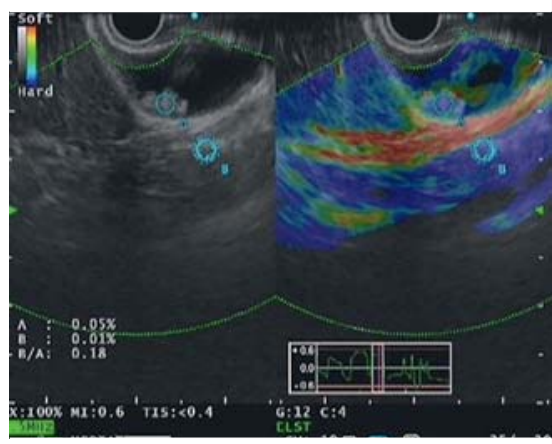

- Fig. 1 Endoscopic ultrasound view of the gallbladder showing a hyperechoic lesion about $1.0 \mathrm{~cm}$ in diameter.
A 52-year-old woman with gallbladder polyps had a strong wish to keep her gallbladder. Abdominal ultrasound showed the contractile function of her gallbladder was good. Imaging including endoscopic ultrasound (EUS), computed tomography (CT), and abdominal ultrasound indicated that these polyps were benign lesions. Therefore, an EUS-guided gallbladder polyp resection was performed, instead of cholecystectomy ( $>$ Video 1).

Firstly, the gallbladder was scanned by EUS at the duodenal bulb, which

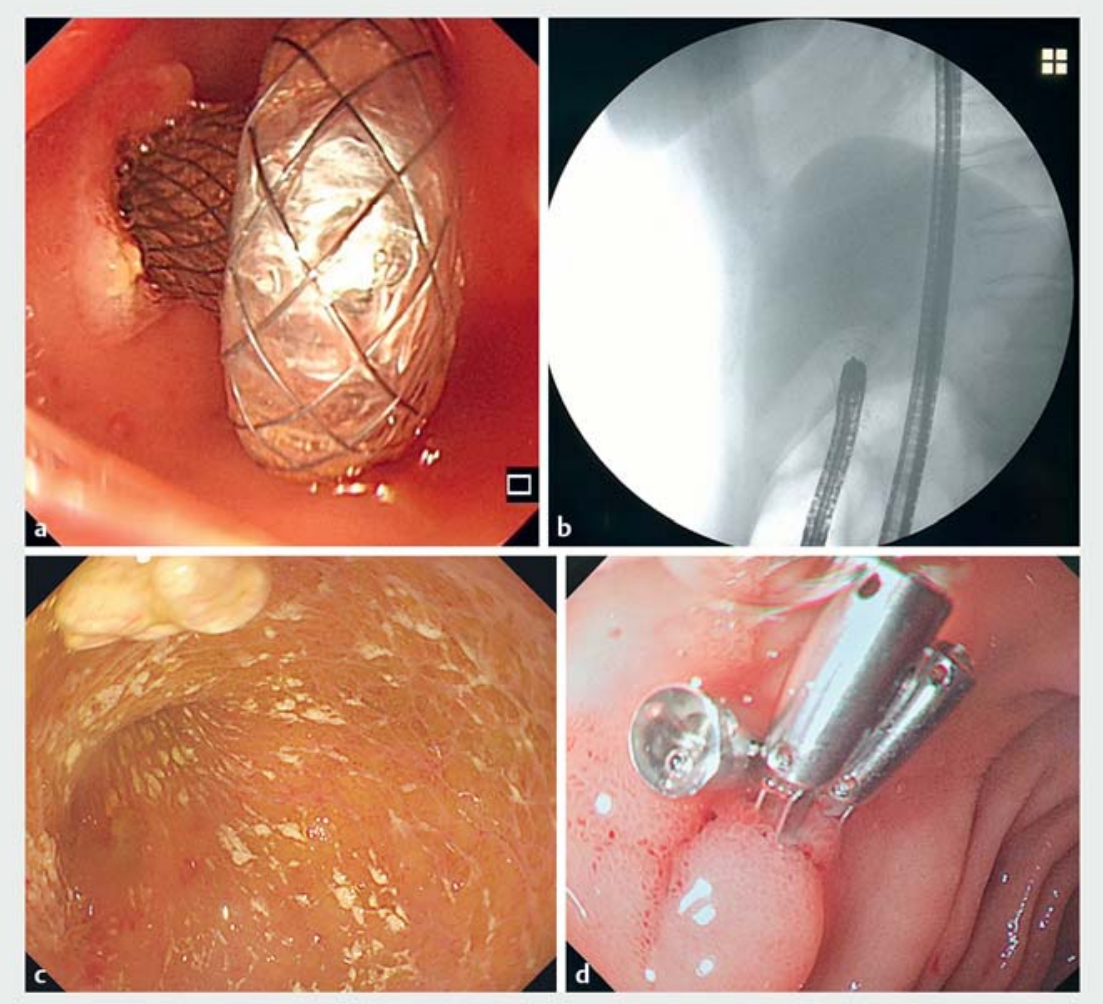

- Fig. 2 The procedure for polyp resection via an endoscopic ultrasound-guided cholecystoduodenostomy using a lumen-apposing metal stent (LAMS). a Endoscopic view showing the LAMS successfully placed with the distal flange deployed in the gallbladder and the proximal flange in the duodenal bulb. b Radiographic image confirming the position of the LAMS. $c$ View using a gastroscope passed through the LAMS showing a gallbladder polyp. d Endoscopic view showing the fistula closed by clips. showed a hyperechoic lesion ( $\mathbf{F i g} \mathbf{1}$ ). A 19-gauge needle was used to puncture the body of the gallbladder and this was followed by injection of contrast medium through the needle. A 0.035 -inch guidewire was then advanced through the needle to allow insertion of an electrocautery-enhanced lumen-apposing metal stent (ECE-LAMS). The LAMS was released and its position was confirmed radiographically ( Fig. 2a,b).

Nasojejunal enteral nutrition was administered. One week after the cholecystoduodenostomy, a gastroscope was introduced into the gallbladder via the LAMS, and three polys were revealed ( $\triangleright$ Fig. 2c), two of which were initially resected by snares. Owing to the limited space and visual field for operating, the LAMS was removed straight after these resections. The last polyp was then resected, and the fistula was immediately closed with clips ( $\triangleright$ Fig. 2 d).

During 2 months of follow-up, the patient recovered well without any complications, such as peritonitis, infection, or bleeding. Pathological examination of the resected polyps indicated that they were adenomatous polyps ( $\triangleright$ Fig. 3 ).

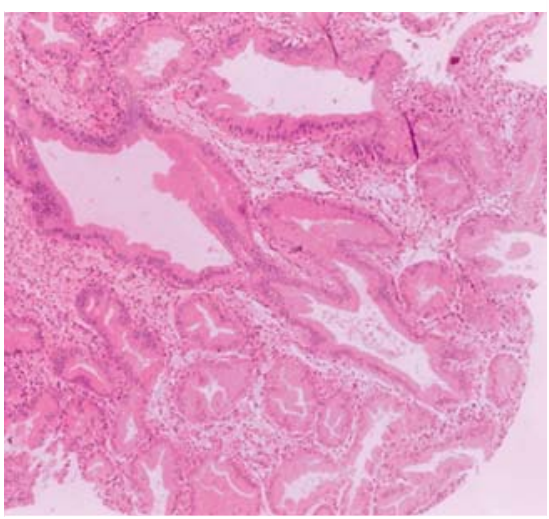

Fig. 3 Pathological examination of the resected polyps showing adenomatous polyps. 


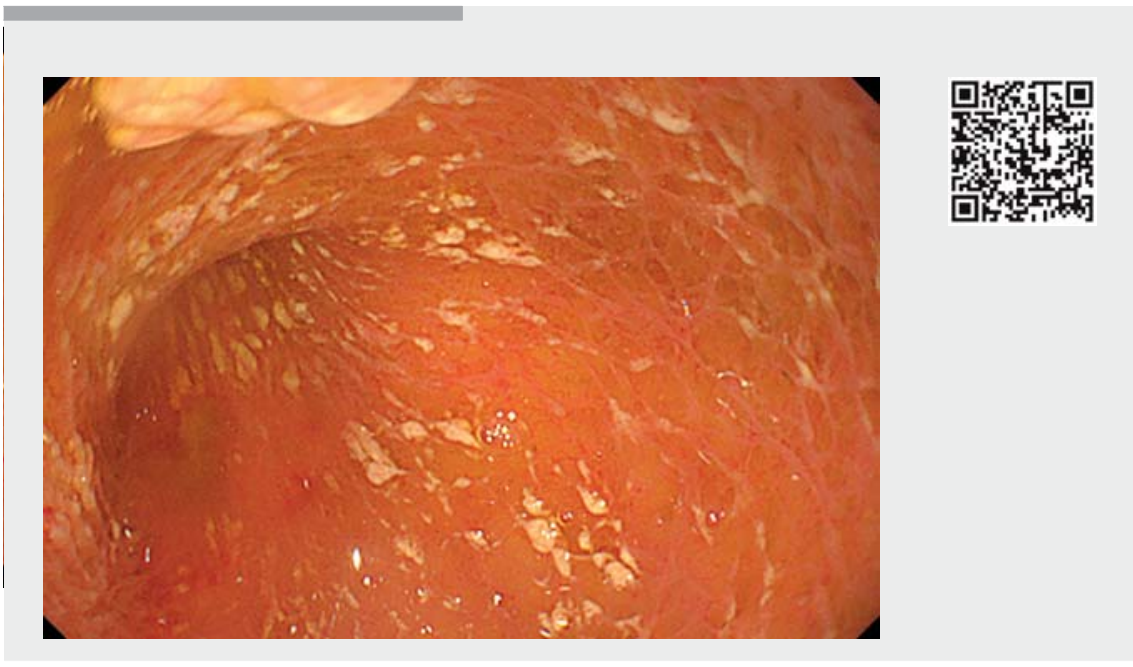

Video 1 Endoscopic ultrasound-guided cholecystoduodenostomy using an electrocautery-enhanced lumen-apposing metal stent for the treatment of gallbladder polyps.

\section{References}

[1] Ge N, Sun S, Sun S et al. Endoscopic ultrasound-assisted transmural cholecystoduodenostomy or cholecystogastrostomy as a bridge for per-oral cholecystoscopy therapy using double-flanged fully covered metal stent. BMC Gastroenterol 2016; 16: 9

[2] Dollhopf M, Larghi A, Will U et al. EUS-guided gallbladder drainage in patients with acute cholecystitis and high surgical risk using an electrocautery-enhanced lumenapposing metal stent device. Gastrointest Endosc 2017; 86: 636-643

\section{Bibliography}

DOI https://doi.org/10.1055/a-0631-7970

Published online: 8.8.2018

Endoscopy 2018; 50: E307-E308

(c) Georg Thieme Verlag KG

Stuttgart · New York

ISSN 0013-726X

This is the first case report to show in detail the procedure of EUS-guided gallbladder polyp resection, thereby avoiding cholecystectomy. Compared with laparoscopic cholecystectomy, the advantage of this endoscopic technique is retention of the gallbladder, which is especially important for those who are diagnosed with gallbladder polyps but have a well-functioning gallbladder [1]. In this case, an ECE-LAMS, a novel stent combining a LAMS with an electrocautery-enhanced delivery system, was used for the formation of the fistula tract [2]. The use of the ECE-LAMS promoted the efficiency and safety of the procedure by reducing the time needed to replace accessories that would otherwise have been required to place the stent.

Endoscopy_UCTN_Code_TTT_1AS_2AG
Competing interests

None

The authors

Li Tian", Yuanyuan Yang", Dinghua Xiao, Rui Wang, Xiayu Li, Shourong Shen, Xiaoyan Wang

Department of Gastroenterology and Hunan Key Laboratory of Nonresolving Inflammation and Cancer, The Third Xiangya Hospital, Changsha, Hunan China

\section{Corresponding author}

\section{Xiaoyan Wang, MD, PhD}

Department of Gastroenterology, The Third Xiangya Hospital, Central South University, Changsha, Hunan 410013, China wxytal2016@163.com

\section{ENDOSCOPY E-VIDEOS}

https://eref.thieme.de/e-videos

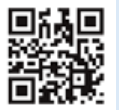

Endoscopy E-Videos is a free access online section, reporting on interesting cases and new techniques in gastroenterological endoscopy. All papers include a high quality video and all contributions are freely accessible online.

This section has its own submission website at https://mc.manuscriptcentral.com/e-videos

\footnotetext{
* Contributed equally to this work
} 